\title{
Direct IR spectroscopic detection of a low-lying electronic state in a metal carbide cluster
}

\author{
Valeriy Chernyy * Remko Logemann ${ }^{\dagger} \quad$ Andrei Kirilyuk ${ }^{\star}$ \\ Joost M. Bakker ${ }^{\text {III }}$
}

February 1, 2018

\section{Abstract}

The electronic structure of metal clusters is notoriously difficult to detect spectroscopically, due to rapid relaxation into the ground state following excitation. We have used IR multiple photon excitation to identify a low-lying electronic state in a tantalum carbide cluster. The electronic excitation is found at 458 $\mathrm{cm}^{-1}$, and is confirmed by experiments on isotopically labeled clusters. Timedependent density functional theory (TD-DFT) calculations confirm the current assignment, but a second predicted electronic state was not observed.

The electronic structure of atoms and molecules is one of the main factors determining their physical and chemical properties. In clusters - particles consisting of a few to several tens or hundreds of atoms - the transition between atomic and bulk regimes of matter is well illustrated by the steady increase in the density of states, initially consisting of discrete electronic states, but gradually merging into a band structure familiar in bulk.

The most direct way to map the electronic structure is through spectroscopic characterization, usually in combination with mass-spectrometric separation of clusters that tend to be produced in distributions of various sizes. For clusters with a net negative charge, mass-selection followed by photodetachment has

\footnotetext{
*Radboud University, Institute for Molecules and Materials, Heyendaalseweg 135, 6525 AJ Nijmegen, the Netherlands

${ }^{\dagger}$ Radboud University, Institute for Molecules and Materials, Heyendaalseweg 135, 6525 AJ Nijmegen, the Netherlands

${ }^{\ddagger}$ Radboud University, Institute for Molecules and Materials, Heyendaalseweg 135, 6525 AJ Nijmegen, the Netherlands

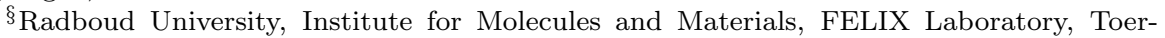
nooiveld 7c, 6525 ED Nijmegen, the Netherlands

`Corresponding author; j.bakker@science.ru.nl; orcid: 0000-0002-1394-7661

${ }$ Radboud University, Institute for Molecules and Materials, FELIX Laboratory, Toernooiveld 7c, 6525 ED Nijmegen, the Netherlands
} 
allowed for the detection of electronic states in the neutral cluster through evaluation of the kinetic energy of the emitted electron. It should be noted, though, that the only neutral states which can be probed in photodetachment spectroscopy are those which are Frank-Condon accessible from the anion geometry, which is not necessarily that of the neutral $[1,2]$.

For neutral clusters, the spectroscopic characterization of electronic structure is complicated by the need to absorb a further photon to ionize and massspectrometrically detect clusters. The possibility to absorb this second photon is hampered by a rapid relaxation of the excited state, especially for clusters containing transition metal atoms, whose partly filled d-shells give rise to a very large number of electronic states. It is perhaps illustrative that conventional spectroscopic techniques to probe electronic structure, such as resonanceenhanced multiphoton ionization - standard for large organic molecules (for instance Gramicidin, $\mathrm{C}_{99} \mathrm{H}_{140} \mathrm{~N}_{20} \mathrm{O}_{17}[3]$ ) - have not been successful for systems larger than metal dimers or trimers [4].

In a recent work, we have studied how the excitation of pure vibrational coordinates can be followed by a relaxation into electronically excited states through vibronic coupling. This way, low-lying electronic states are revealed by the appearance of extra structure in the photoionization spectrum $[5,6]$. The electronic states had energies of approx. $0.3-0.5 \mathrm{eV}$ above the ground state, and their detection efficiency was consistent with a thermal occupation, requiring the absorption of a large number of IR photons. Of course, the reverse of this process, dissipation of electronic excitation energy by vibrational coordinates is widely known, for instance as the origin of IR emission by interstellar molecules $[7,8]$. It is thus not unreasonable to expect that purely electronic transitions could be detected, having the same signature as vibrational transitions. Here, we report such an observation, namely the direct detection of a low-lying electronic state.

We detected this low-lying electronic state in the neutral $\mathrm{Ta}_{5} \mathrm{C}_{3}$ tantalum carbide cluster through IR-UV spectroscopy in the $300-800 \mathrm{~cm}^{-1}$ IR spectral range. The experimental instrument shown in Figure S1 was described in detail in Ref. [9]. Briefly, TaC clusters are produced by laser ablation of solid tantalum in the presence of helium seeded with $1 \%$ methane, similar to previous studies on these clusters $[10,11]$. The carrier gas is pulsed into a clustering channel allowing for tantalum carbide formation until the mixture of clusters and carrier gas expands into vacuum, forming a molecular beam. The beam is collimated by a $2 \mathrm{~mm}$ skimmer before being further shaped by a $1 \mathrm{~mm}$ diameter aperture. Before the aperture, all charged species are deflected out of the beam by two electrically biased plates.

The clusters are irradiated by an IR laser pulse produced by the FELIX free-electron laser [12] based at Radboud University, Nijmegen, the Netherlands, before being ionized by a ns duration pulse of a frequency-doubled dye laser. It is important to note that the IR pulse consists of a $12 \mu$ s duration pulse train of transform limited ps duration pulses, with a 1 ns time separation. This pulse structure has in the past been a decisive factor in efficiently pumping of vibrations [13], thereby allowing the time in between pulses for an 
energy transfer into the vibrational bath assisted by the intramolecular vibrational relaxation (IVR). TaC clusters are known to exhibit a strong coupling between vibrational and electronic systems which can, if a vibration is pumped with sufficient IR light, result in thermionic emission [11]. We here attenuate the IR laser sufficiently to suppress all direct ionization. The wavelength of the probe laser is tuned to $258 \mathrm{~nm}(4.80 \mathrm{eV} /$ photon $)$. This is chosen close to, but still below the photoionization threshold of $4.81 \pm 0.05 \mathrm{eV}$ reported $\mathrm{Ta}_{5} \mathrm{C}_{3}$ [14]. The UV pulse energy is reduced to $100-120 \mu \mathrm{J}$, in order to avoid extensive multiphoton ionization. After interaction with both lasers, all ions formed are extracted into a reflectron time-of-flight mass spectrometer with a mass resolution $M / \Delta M \approx 1800$ and registered on a multi channel plate detector. The experiment is operated at twice the FELIX frequency, allowing to record mass spectra with and without IR excitation in a shot-to-shot manner.

The experimental IR spectrum for $\mathrm{Ta}_{5} \mathrm{C}_{3}$ is depicted in Figure 1(a). The data are presented as the gain, defined as the normalized difference of ions detected with $\left(I_{\mathrm{IR}+\mathrm{UV}}(\omega)\right)$ and without $\left(I_{\mathrm{UV}}\right)$ IR radiation as

$$
G(\omega)=\frac{I_{\mathrm{IR}+\mathrm{UV}}(\omega)-I_{\mathrm{UV}}}{I_{\mathrm{UV}}},
$$

at IR frequency $\omega$. The spectrum is shown in two parts, where the 100-350 $\mathrm{cm}^{-1}$ region is scaled to enhance visibility of the structure. This part was recorded at 2.5-16.4 mJ IR pulse energies, while in the $350-900 \mathrm{~cm}^{-1}$ region the pulse energies range from 0.04 to $0.09 \mathrm{~mJ}$. Both parts of the spectrum are IR power corrected. In the low-energy one, we distinguish at least 3 peaks with maxima at 137, 156 and $192 \mathrm{~cm}^{-1}$. In the high-energy region, we observe maxima at 458, 570,610, 667, 742 and $854 \mathrm{~cm}^{-1}$.

To interpret the spectra, we carried out Density Functional Theory (DFT) calculations. To master the wealth of possible geometric structures, we employed a genetic algorithm (GA) [15] that controls the Vienna ab-initio simulation package (VASP) [16], and found three lowest in energy candidates. To verify the structures are true minima and to compare to the experimental spectra, the selected isomers were further optimized in VASP, and the harmonic frequencies were calculated. Clusters are placed in a 20 Åperiodic box, and standard recommended projector augmented wave (PAW) potentials $[17,18]$ with an energy cutoff of $400.0 \mathrm{eV}$ and a single $\mathbf{k}$ point $(\Gamma)$ are used. All forces were minimized to below $10^{-3} \mathrm{eV} / \AA$. Since bulk $\mathrm{TaC}$ is metallic and superconducting at low temperatures [19] we use the Perdew, Burke and Ernzerhof (PBE) functional [20]. As can be seen in the supplementary materials, the comparison between experimental and theoretical spectra using different functionals confirms that the $\mathrm{Ta}_{5} \mathrm{C}_{3}$ cluster is best described by the PBE functional.

The calculated IR spectra for the three lowest-energy isomers are presented in Figure 1(b-d). Isomers are characterized with their relative energies with respect to the lowest-energy structure (isomer $\mathbf{A}$ ), taking into account the zeropoint vibrational energy. The vibrational frequencies presented are unscaled and depicted by red sticks, as well as by a convolution (blue) with a Gaussian line shape function with a $15 \mathrm{~cm}^{-1}$ width (FWHM). 
All three lowest-energy isomers found for $\mathrm{Ta}_{5} \mathrm{C}_{3}$ are based on a trigonal $\mathrm{Ta}_{5}$ bipyramid, whose equatorial plane is represented by an isosceles triangle. The triangle does not act as a mirror plane for the bipyramid as the two pyramids that it separates are not identical. However, a mirror plane perpendicular to the equatorial plane is present for all the isomers and results in a $\mathrm{C}_{s}$ point group symmetry for each structure.

The lowest-energy isomer $\mathbf{A}$ found here confirms previously reported results [14] and consists of one pyramid where each face is capped with a $\mathrm{C}$ atom, while the other pyramid remains uncapped. Structures $\mathbf{B}$ and $\mathbf{C}$ are found at substantially higher energies $(+1.320 \mathrm{eV}$ and $+1.323 \mathrm{eV}$ with respect to $\mathbf{A}$, respectively) and have a more spread-out distribution of the $\mathrm{C}$ atoms. Therefore, the energetics of the calculated structures already heavily favor structure $\mathbf{A}$. Additionally, the comparison between calculated and experimental vibrational spectra also points at the presence of this structure. Each of the seven experimental resonances indicated by dashed lines are also found in the calculated vibrational spectra (blue) of $\mathbf{A}$, and none of the pronounced calculated resonances is missing. One would thus be tempted to assign the spectrum of $\mathrm{Ta}_{5} \mathrm{C}_{3}$ directly to structure $\mathbf{A}$, were it not for the looming presence of the pronounced band centered at $458 \mathrm{~cm}^{-1}$.

If not vibrational, what is then the origin of this band? For this, we note that in our earlier work on niobium carbide clusters, we found low-lying electronic states at $0.35 \mathrm{eV}[6]$, and that the $\mathrm{TaC}$ dimer anion exhibits an electronic state at $0.362 \mathrm{eV}[21]$. It is thus not unreasonable to suspect that $\mathrm{Ta}_{5} \mathrm{C}_{3}$ can also exhibit such low-lying states. The energies of the Kohn-Sham orbitals for isomer A obtained employing the ground-state DFT calculations strengthens our hypothesis.

To investigate this aspect computationally, we employed linear response time-dependent DFT (TD-DFT), a perturbative extension of regular DFT for time-dependent, oscillating external potentials [22, 23], to calculate the twenty lowest electronically excited states and their associated oscillator strength. This method has been demonstrated to yield satisfactory electronic excitation spectra for finite systems [24]. We used TD-DFT as implemented in ADF2016 [25, 26], at the PBE/QZ4P level (no frozen core), using the zeroth order regular approximation to account for the relativistic effects. Since ADF uses Slater-type orbitals rather than plane waves as basis, each isomer was re-optimized. The geometry re-optimization resulted in an average difference in coordinates of less than $0.006 \AA$ and a $0.5 \mathrm{meV}$ difference in the lowest electronic excitation energy. To ensure consistency, we recalculated the vibrational spectrum, which was essentially identical to the VASP calculated one.

The excitations found are displayed in Figure 3, and a surprisingly good match is found for the lowest $A^{\prime}$ excitation, at $59 \mathrm{meV}$ or $476 \mathrm{~cm}^{-1}$, comparing very favorably with the experimentally found $458 \mathrm{~cm}^{-1}$. The electronic transition has an oscillator strength of $2.8 \cdot 10^{-4}$, and the corresponding transition dipole moment is oriented perpendicular to the equatorial $\mathrm{Ta}_{3}$ plane. Note that we also converted the oscillator strength into the absorptivity which we plot on the same scale with the vibrational transitions in Figure 1 (b), in green. 
To experimentally verify the hypothesis that the $458 \mathrm{~cm}^{-1}$ resonance corresponds to an electronic transition, we used isotopic substitution of the carbon atoms to study a possible frequency shift of the band. By seeding the helium carrier gas with a 1:1 mixture of ${ }^{12} \mathrm{CH}_{4}$ and ${ }^{13} \mathrm{CH}_{4}$ we produce a 1:3:3:1 distribution of $\mathrm{Ta}_{5}{ }^{12} \mathrm{C}_{3}: \mathrm{Ta}_{5}{ }^{12} \mathrm{C}_{2}{ }^{13} \mathrm{C}_{\mathrm{Ta}}{ }_{5}{ }^{12} \mathrm{C}^{13} \mathrm{C}_{2}: \mathrm{Ta}_{5}{ }^{13} \mathrm{C}_{3}$, as is shown on panel (c) of Figure 2. By creating this isotopic mixture, a simultaneous measurement of the vibrational spectra of $\mathrm{Ta}_{5} \mathrm{C}_{3}$ with ${ }^{12} \mathrm{C}$ and its fully substituted ${ }^{13} \mathrm{C}$ isotopologue is enabled, reducing uncertainties in calibration. The simultaneous measurement is at the expense of the number of produced clusters per carbon isotopologue. To compensate for this, we increased the IR laser pulse energy to 0.3-0.4 mJ, which could lead to a slight broadening of the observed bands, but does not change the peak center position. We fit the most pronounced peaks to a Gaussian line shape function (indicated by the thick solid lines in Figure 2 (a)) to determine the band centers. From this procedure, we find that the three highest energy bands shift roughly by $20-30 \mathrm{~cm}^{-1}$, while the $458 \mathrm{~cm}^{-1}$ band only exhibits a negligible shift. This confirms the electronic nature of the band as the electronic transition is in first approximation insensitive to isotopic substitution. For completeness, we also calculated the electronic energy transition for $\mathrm{Ta}_{5}{ }^{13} \mathrm{C}_{3}$ and found it to be identical to that for $\mathrm{Ta}_{5}{ }^{12} \mathrm{C}_{3}$.

It is of course not a surprise that cluster systems, with their wealth of possible electronic configurations, exhibit low-lying states. However, to the best of our knowledge, this is the lowest directly detected electronic state. Moreover, the method of detection, i.e., through IR-multiple photon excitation appears to be the key. If the IR excitation frequency is fixed, and the UV photon energy is modified, a rather broad enhancement in gain is observed (Figure S4), regardless of whether electronic $\left(458 \mathrm{~cm}^{-1}\right)$ or vibrational $\left(742 \mathrm{~cm}^{-1}\right)$ bands are excited. Both of these UV spectra are similar to the curves presented earlier for clusters excited vibrationally $[5,6]$. This points at a statistical redistribution of the energy over the vibrational states absorbed in the electronic excitation detected in this work.

Closer inspection of Figure 3 reveals a second electronic state $(A ")$ that is within the spectral range covered by FELIX. Observation of this second line would confirm the assignment to an electronic state beyond any possible doubt. Unfortunately, this observation was not done, as shown in the SI. Before starting to doubt the accuracy of the used DFT functionals, one could also speculate the mechanism underlying the detection of the pure vibrational bands. Here, the choice of probe wavelength plays a role: by tuning it just below the ionization threshold, it either probes population in excited vibrational states ("hot bands") or population in an excited electronic state. If the latter were the case, it is likely the same energy level as predicted at $458 \mathrm{~cm}^{-1}$, implying a population transfer from (the bath of) vibrational states. One can now wonder whether the coupling between the vibrational states to different electronic states is the same; given the missing electronic band at higher frequencies, we speculate that this coupling may simply be not as strong as for the low-lying state.

Conversely, one can also wonder whether it is the closeness of the low-lying electronic state to the "bright" vibrational states leads to an enhanced vibronic 
coupling. While we are unable to predict this strength, or its consequences, we do note that in our experiments to probe vibrational bands of $\mathrm{Ta}_{5} \mathrm{C}_{3}$ we used IR pulse energies that were an order of magnitude lower than required for observation of the vibrations for niobium carbide clusters [6, 27]), even though the calculated IR intensities are similar. We believe that the possibility to use such a low pulse energy in the case of $\mathrm{Ta}_{5} \mathrm{C}_{3}$ is enabled by the presence of the low-lying electronic state, providing an enhanced intramolecular energy redistribution process.

In conclusion, we performed spectroscopic investigations of the $\mathrm{Ta}_{5} \mathrm{C}_{3}$ cluster in the far infrared spectral region, and have unequivocally observed a direct transition to a low-lying electronic state. This is evidenced by excellent agreement between calculated and experimental vibrational spectra for the lowest energy calculated isomer, as well as by experiments on ${ }^{13} \mathrm{C}$ labeled $\mathrm{Ta}_{5} \mathrm{C}_{3}$ clusters. During the investigations, the electronic band exhibited no shift, while for the observed vibrational bands the predicted change in frequencies was in an excellent agreement with theory, although a second electronic band was not observed.

\section{Acknowledgment}

We gratefully acknowledge the Netherlands Organization for Scientific Research Physical Sciences (NWO) for their financial contribution, including support of the FELIX Laboratory. We also thank NWO and the SurfSara Supercomputer Center for providing the computational resources.

\section{Supplementary Information}

In the supplementary material we present the optimized coordinates and harmonic frequencies of the studied structures, the raw spectra showing the data reduction, experimental UV gain spectra, and experimental results attempting to localize the second predicted electronic transition.

\section{References}

[1] O. Cheshnovsky, K. Taylor, J. Conceicao, and R. E. Smalley. Phys. Rev. Lett., 64(15):1785-1788, 1990.

[2] C Bartels, C Hock, J Huwer, R Kuhnen, J Schwobel, and B von Issendorff. Science, 323(5919):1323-1327, 2009.

[3] Ali Abo-Riziq, Bridgit O. Crews, Michael P. Callahan, Louis Grace, and Mattanjah S. de Vries. Angew. Chem. Int. Ed., 45(31):5166-5169, 2006.

[4] Michael D. Morse. Chem. Rev., 86(6):1049-1109, 1986. 
[5] Jeroen Jalink, Joost M. Bakker, Theo Rasing, and Andrei Kirilyuk. J. Phys. Chem. Lett., 6:750-754, 2015.

[6] V. Chernyy, R. Logemann, J. M. Bakker, and A. Kirilyuk. J. Chem. Phys., 145:024313, 2016.

[7] A Léger and J L Puget. Astron. Astrophys. Suppl. Ser., 137(1):L5-L8, 1984.

[8] L J Allamandola, A.G.G.M. Tielens, and J R Barker. Astrophys. J., 290:L25-L28, 1985.

[9] Florent Calvo, Yejun Li, Denis M Kiawi, Joost M. Bakker, Pascal Parneix, and Ewald Janssens. Phys. Chem. Chem. Phys., 17(39):25956-25967, 2015.

[10] S. Wei, B. C. Guo, J. Purnell, S A Buzza, and A.W. Castleman. Science, 256(5058):818-820, 1992.

[11] Deniz van Heijnsbergen, André Fielicke, Gerard Meijer, and Gert von Helden. Phys. Rev. Lett., 89(1):013401, 2002.

[12] D. Oepts, A.F.G. van der Meer, and P.W. van Amersfoort. Infrared Phys. 86 Techn., 36:297-308, 1995.

[13] Jos Oomens, Gerard Meijer, and Gert von Helden. J. Phys. Chem. A, 105(36):8302-8309, 2001.

[14] Viktoras Dryza, Jason F. Alvino, and Gregory F. Metha. J. Phys. Chem. A, 114(12):4080-4085, 2010.

[15] Roy L. Johnston. Dalton Transactions, 22:4193, 2003.

[16] G. Kresse and J. Furthmüller. Phys. Rev. B, 54(16):11169-11186, 1996.

[17] P. E. Blöchl. Phys. Rev. B, 50(24):17953-17979, 1994.

[18] G. Kresse. Phys. Rev. B, 59(3):1758-1775, 1999.

[19] A. L. Giorgi, E. G. Szklarz, E. K. Storms, Allen L. Bowman, and B. T. Matthias. Phys. Rev., 125:837-838, 1962.

[20] John P. Perdew, Kieron Burke, and Matthias Ernzerhof. Phys. Rev. Lett., 77(18):3865-3868, 1996.

[21] G. Aravind, M. Nrisimhamurty, Rupali G. Mane, A. K. Gupta, and E. Krishnakumar. Phys. Rev. A, 92(4):042503, 2015.

[22] Erich Runge and E. K. U. Gross. Phys. Rev. Lett., 52:997-1000, 1984.

[23] Miguel A L Marques, Neepa T Maitra, Fernando M S Nogueira, Eberhard K U Gross, and Angel Rubio. Lecture Notes in Physics. Springer, Berlin, 2012. 
[24] Giovanni Onida, Lucia Reining, and Angel Rubio. Rev. Mod. Phys., 74:601$659,2002$.

[25] G. te Velde, F. M. Bickelhaupt, E. J. Baerends, C. Fonseca Guerra, S. J. A. van Gisbergen, J. G. Snijders, and T. Ziegler. J. Comp. Chem., 22:931-967, 2001.

[26] C. Fonseca Guerra, J. G. Snijders, G. te Velde, and E. J. Baerends. Theor. Chim. Acta, 99:391-403, 1998.

[27] V. Chernyy, R. Logemann, J. M. Bakker, and A. Kirilyuk. J. Chem. Phys., 145(16):164305, 2016. 


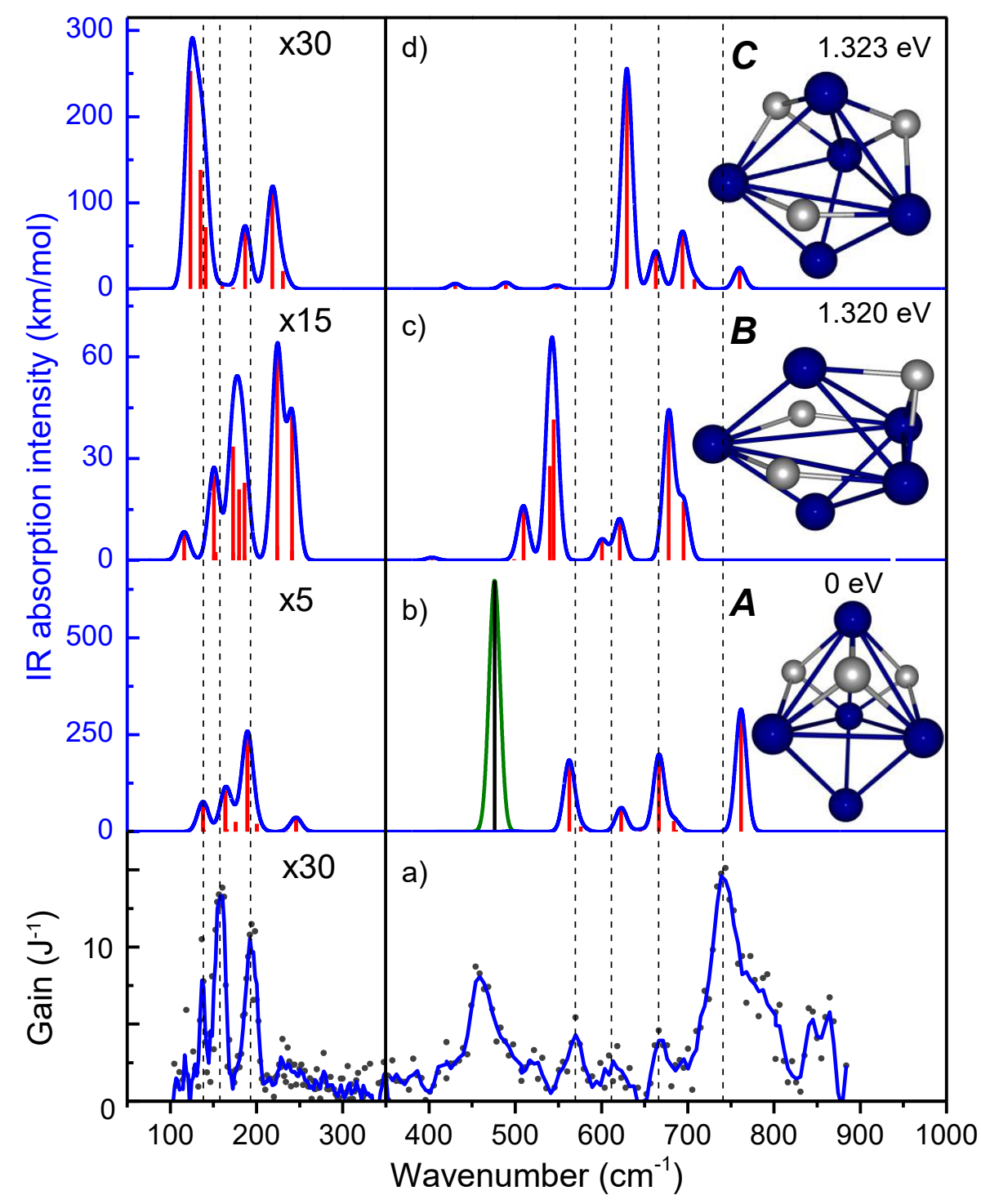

Figure 1: a) - experimental IR spectrum for $\mathrm{Ta}_{5} \mathrm{C}_{3}$ (circles) accompanied by a three-point adjacent average (blue line). (b-d) - vibrational frequencies (red sticks) calculated for different $\mathrm{Ta}_{5} \mathrm{C}_{3}$ isomers (insets) convoluted with a $15 \mathrm{~cm}^{-1}$ FWHM Gaussian line shape function (blue). Green - calculated electronic transition. Color coding corresponding geometries: C-gray, Ta-blue. 


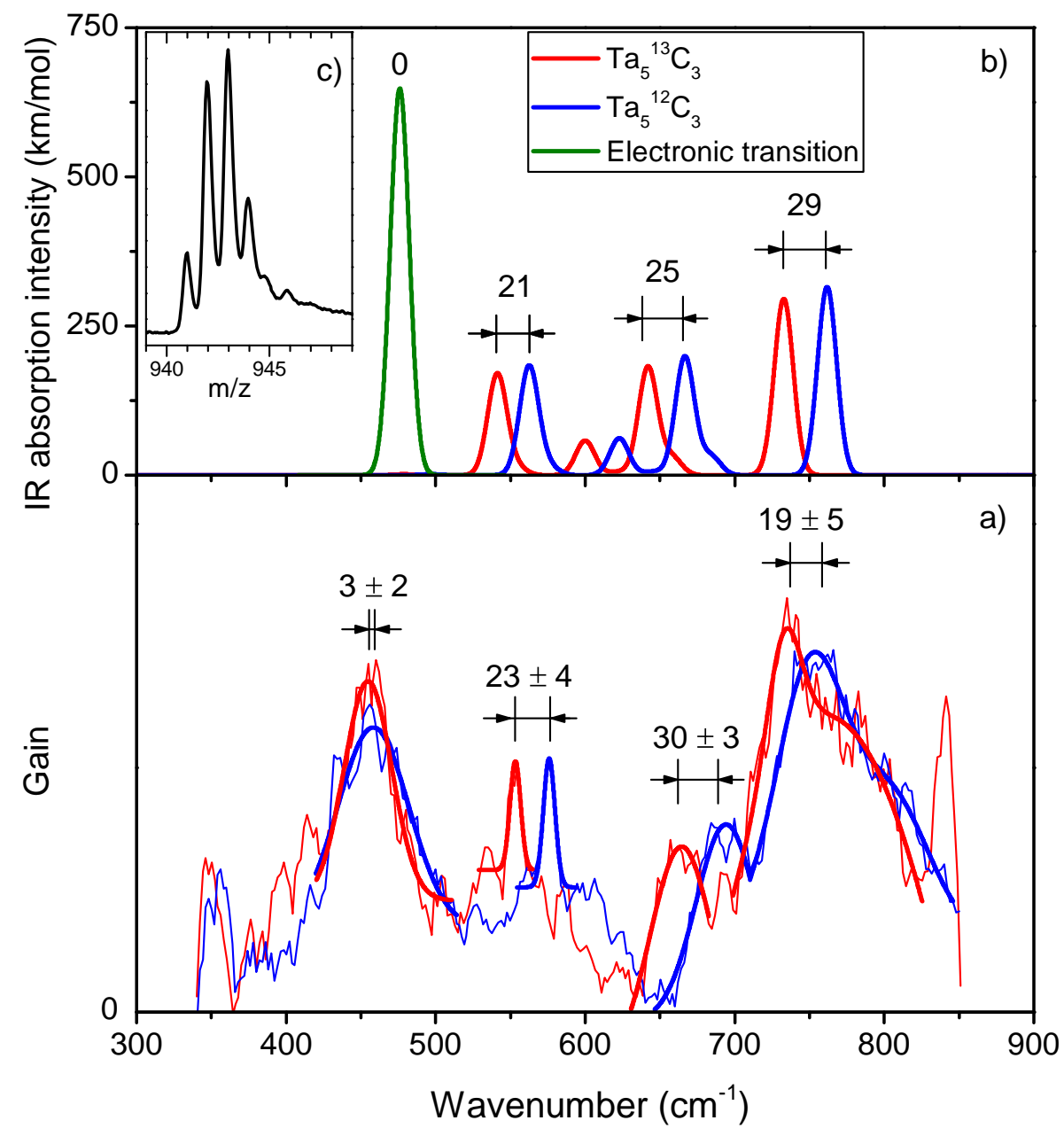

Figure 2: Experimental (a) and calculated (b) IR spectra for $\mathrm{Ta}_{5}{ }^{12} \mathrm{C}_{3}$ (blue) and $\mathrm{Ta}_{5}{ }^{13} \mathrm{C}_{3}$ (red). Numbers indicate the experimental and calculated isotopic shifts $\left(\right.$ in $\mathrm{cm}^{-1}$ ). The fits of the most intense peaks with the Gaussian line shape functions are shown by thick lines. (c) - mass spectrum; peaks corresponding cluster sizes (from left to right): $\mathrm{Ta}_{5}{ }^{12} \mathrm{C}_{3}$ : $\mathrm{Ta}_{5}{ }^{12} \mathrm{C}_{2}{ }^{13} \mathrm{C}_{\mathrm{Ta}}{ }^{12} \mathrm{C}^{13} \mathrm{C}_{2}$ :Ta ${ }^{13} \mathrm{C}_{3}$. 


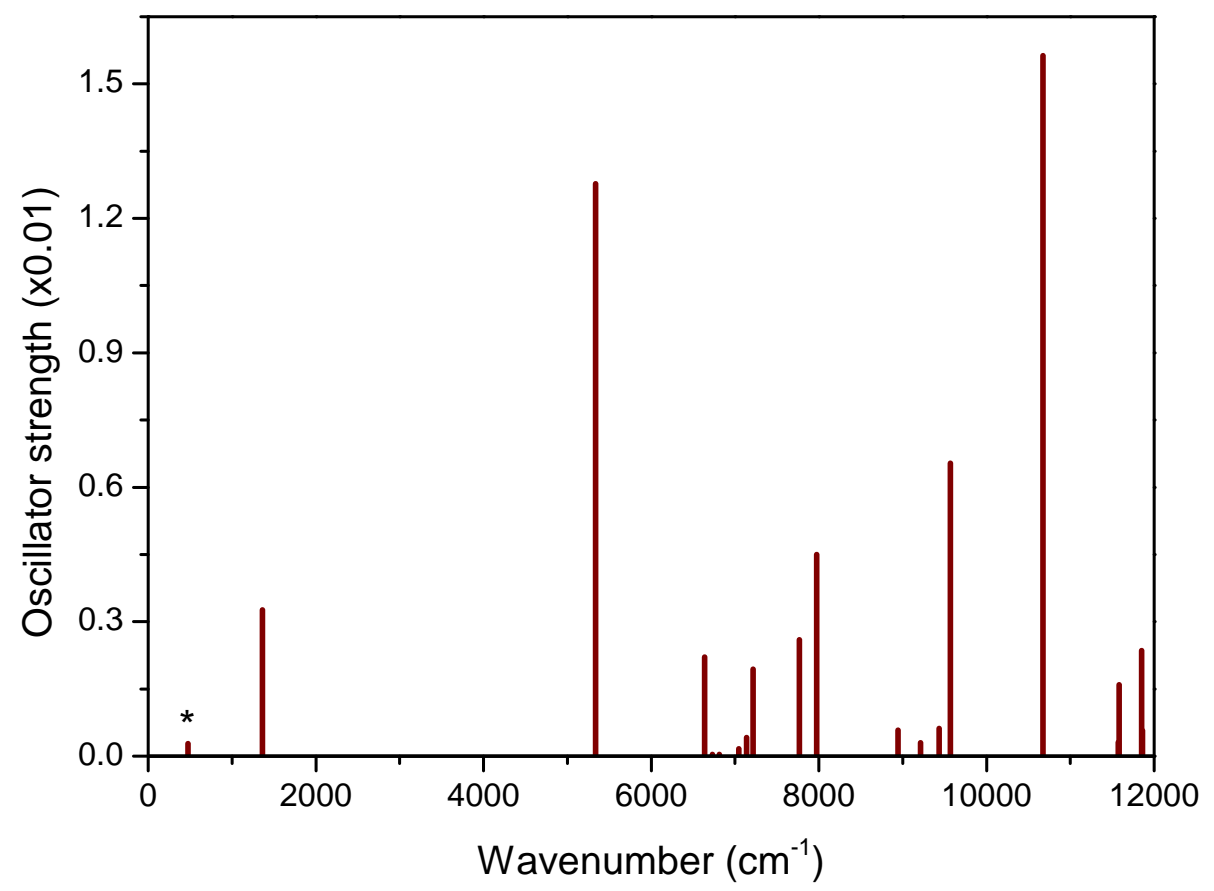

Figure 3: TD-DFT calculated electronic excitation energies for $\mathrm{Ta}_{5} \mathrm{C}_{3}$, isomer A. The experimentally observed electronic excitation at $59 \mathrm{meV}$ is marked by an asterisk.

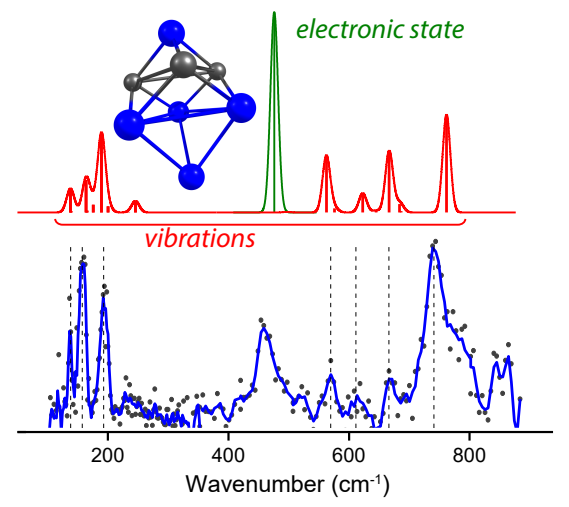

Figure 4: TOC figure. 